TRANSACTIONS OF THE

AMERICAN MATHEMATICAL SOCIETY

Volume 360, Number 9, September 2008, Pages 4619-4638

S 0002-9947(08)04295-5

Article electronically published on April 11, 2008

\title{
LOW REGULARITY GLOBAL WELL-POSEDNESS FOR THE ZAKHAROV AND KLEIN-GORDON-SCHRÖDINGER SYSTEMS
}

\author{
JAMES COLLIANDER, JUSTIN HOLMER, AND NIKOLAOS TZIRAKIS
}

\begin{abstract}
We prove low regularity global well-posedness for the 1d Zakharov system and the 3d Klein-Gordon-Schrödinger system, which are systems in two variables $u: \mathbb{R}_{x}^{d} \times \mathbb{R}_{t} \rightarrow \mathbb{C}$ and $n: \mathbb{R}_{x}^{d} \times \mathbb{R}_{t} \rightarrow \mathbb{R}$. The Zakharov system is known to be locally well-posed in $(u, n) \in L^{2} \times H^{-1 / 2}$ and the Klein-GordonSchrödinger system is known to be locally well-posed in $(u, n) \in L^{2} \times L^{2}$. Here, we show that the Zakharov and Klein-Gordon-Schrödinger systems are globally well-posed in these spaces, respectively, by using an available conservation law for the $L^{2}$ norm of $u$ and controlling the growth of $n$ via the estimates in the local theory.
\end{abstract}

\section{INTRODUCTION}

The initial-value problem for the one-dimensional Zakharov system is

$$
\left\{\begin{array}{l}
i \partial_{t} u+\partial_{x}^{2} u=n u, \\
\partial_{t}^{2} n-\partial_{x}^{2} n=\partial_{x}^{2}|u|^{2} \\
u(x, 0)=u_{0}(x), n(x, 0)=n_{0}(x), \partial_{t} n(x, 0)=n_{1}(x) .
\end{array}\right.
$$

Here $u:\left[0, T^{*}\right) \times \mathbb{R} \longmapsto \mathbb{R}, n:\left[0, T^{*}\right) \times \mathbb{R} \longmapsto \mathbb{R}$. This problem arises in plasma physics. Sufficiently regular solutions of (1.1) satisfy conservation of mass

$$
M[u](t)=\int|u(t)|^{2} d x=\int\left|u_{0}\right|^{2} d x=M\left[u_{0}\right]
$$

and conservation of the Hamiltonian

$$
\begin{aligned}
H[u, n, \nu](t) & =\int\left(\left|\partial_{x} u(t)\right|^{2}+\frac{1}{2} n(t)^{2}+n(t)|u(t)|^{2}+\frac{1}{2} \nu(t)^{2}\right) d x \\
& =H\left[u_{0}, n_{0}, \nu_{0}\right]
\end{aligned}
$$

where $\nu(t)$ is such that $\partial_{t} n=\partial_{x} \nu$ and $\partial_{t} \nu=\partial_{x}\left(n+|u|^{2}\right)$.

The local-in-time theory in $X_{s, b}$ spaces has been established in 4, 11, the latter paper obtaining local well-posedness (LWP) for the one-dimensional equation (1.1) with $\left(u_{0}, n_{0}, n_{1}\right) \in L^{2} \times H^{-1 / 2} \times H^{-3 / 2}$ and for some more regular spaces

Received by the editors March 27, 2006 and, in revised form, April 17, 2006.

2000 Mathematics Subject Classification. Primary 35Q55.

Key words and phrases. Zakharov system, Klein-Gordon-Schrödinger system, global wellposedness.

The first author was partially supported by N.S.E.R.C. Grant RGPIN 250233-03 and the Sloan Foundation.

The second author was supported by an NSF postdoctoral fellowship.

(C)2008 American Mathematical Society 
$H^{k} \times H^{s} \times H^{s-1}$ with varioun $1, s$. As an immediate consequence of the local theory and (1.2), (1.3), one has global well-posedness (GWP) for $k=1, s=0$. Pecher [16], using the low-high frequency decomposition method of Bourgain [6], proved GWP for $\frac{9}{10}<k<1, s=0$. This result was improved in 18] using the $I$-method of [8] to obtain GWP for $\frac{5}{6}<k<1, s=k-1$. The preceding GWP results are all based on the conservation of the Hamiltonian (1.3) or certain variants of the Hamiltonian. In this paper, we prove GWP for $k=0, s=-\frac{1}{2}$, using a scheme based on mass conservation (1.2) and subcritical slack in certain multilinear estimates at this regularity threshold. In [12, it is shown that the onedimensional LWP theory of [11] is effectively sharp by adapting techniques of [5] and 77. Thus, we establish GWP in the largest space for which LWP holds.

Theorem 1.1. The Zakharov system (1.1) is globally well-posed for $\left(u_{0}, n_{0}, n_{1}\right) \in$ $L^{2} \times H^{-1 / 2} \times H^{-3 / 2}$, and the solution $(u, n)$ satisfies (1.2) and

$$
\|n(t)\|_{H_{x}^{-\frac{1}{2}}}+\left\|\partial_{t} n(t)\right\|_{H_{x}^{-\frac{3}{2}}} \leq \exp \left(c|t|\left\|u_{0}\right\|_{L^{2}}^{2}\right) \max \left(\left\|n_{0}\right\|_{H^{-\frac{1}{2}}}+\left\|n_{1}\right\|_{H^{-\frac{3}{2}}},\left\|u_{0}\right\|_{L^{2}}^{2}\right) .
$$

Remark 1.2. Since Theorem 1.1 is based on the mass conservation property (1.2) and local theory, the same result applies to certain Hamiltonian generalizations of (1.1) for which global well-posedness was previously unknown. Indeed, if we write

$$
H[u, n, \nu](t)=\int\left|\partial_{x} u(t)\right|^{2}+\frac{\alpha}{2}|n(t)|^{2}+\frac{\beta}{2}|\nu(t)|^{2}+\gamma n(t)|u(t)|^{2} d x
$$

and calculate

$$
\partial_{t}\left[\begin{array}{l}
u \\
n \\
\nu
\end{array}\right]=\left[\begin{array}{ccc}
-i & 0 & 0 \\
0 & 0 & \nabla \cdot \\
0 & \nabla & 0
\end{array}\right]\left[\begin{array}{l}
H_{\bar{u}} \\
H_{n} \\
H_{\nu}
\end{array}\right]
$$

we find the evolution system

$$
\left\{\begin{array}{l}
i \partial_{t} u+\partial_{x}^{2} u=\gamma n u \\
\partial_{t}^{2} n-\alpha \beta \partial_{x}^{2} n=\beta \gamma \partial_{x}^{2}|u|^{2}
\end{array}\right.
$$

If we then choose $\alpha=\beta=-1$ and $\gamma=-1$, we encounter a Hamiltonian evolution problem similar to (1.1) but with $+n u$ replaced by $-n u$. The local theory for these problems coincides, but the appearance of $\alpha=\beta=-1$ in the Hamiltonian $H$ precludes its use in obtaining a globalizing estimate.

The initial-value problem for the $d$-dimensional Klein-Gordon-Schrödinger system with Yukawa coupling is

$$
\left\{\begin{array}{l}
i \partial_{t} u+\Delta u=-\gamma n u, \\
\partial_{t}^{2} n+\alpha \beta(1-\Delta) n=-\beta \gamma|u|^{2}, \\
u(x, 0)=u_{0}(x), n(x, 0)=n_{0}(x), \partial_{t} n(x, 0)=n_{1}(x) .
\end{array} \quad x \in \mathbb{R}\right.
$$

\footnotetext{
${ }^{1}$ The paper [11 actually gives a systematic treatment of LWP for higher dimensional versions of (1.1) as well. Their result in dimension one uses the calculus techniques for obtaining $X_{s, b}$ bilinear estimates developed by Kenig, Ponce and Vega 14], 15]. The only LWP result in [11] when $k=0$ is for dimension one, $s=-\frac{1}{2}$, and thus we have restricted ourselves exclusively to this case.

${ }^{2}$ Although the additional assumption $n_{1} \in \dot{H}^{-1}$ appears in the papers [16] and [18], it can likely be removed by introducing suitable low frequency modifications to the energy identity; see 10] Lemma A.1, p. 358.
} 
Here $\alpha, \beta, \gamma$ are real constants. The solution satisfies conservation of mass

$$
M[u](t)=\int|u(t)|^{2} d x=\int\left|u_{0}\right|^{2} d x=M\left[u_{0}\right]
$$

and conservation of the Hamiltonian

$$
\begin{aligned}
H[u, n, \nu](t) & =\int\left(|\nabla u(t)|^{2}+\frac{1}{2 \beta}\left|\partial_{t} n(t)\right|^{2}+\frac{\alpha}{2}|\sqrt{-\Delta+1} n(t)|^{2}+\gamma n(t)|u(t)|^{2}\right) d x \\
& =H\left[u_{0}, n_{0}, \nu_{0}\right] .
\end{aligned}
$$

Pecher 17] proved that (1.4) is LWP for $d=3$ in $L^{2} \times L^{2} \times H^{-1}$ and some more regular spaces $H^{k} \times H^{s} \times H^{s-1}$ for various $k, s$ by following the scheme developed for the Zakharov system in [11. Provided that $\alpha>0$ and $\beta>0$, energy conservation (1.6) yields GWP in the setting $1 \leq d \leq 3$ and $k=1, s=1$. In the case when $\alpha>0$ and $\beta>0$ where the energy gives control on the $H^{1}$ norm, the low-high frequency separation method of Bourgain [6] has been applied to (1.4) in [17] and the method of almost conservation laws of 8 has been applied to (1.4) in [22, to obtain GWP under the following assumptions: for $d=1, k=s, s>\frac{1}{2}$; for $d=2$, $k=s, s>\frac{\sqrt{17}-3}{2}$; for $d=3, k=s, s>\frac{7}{10}$; for $d=3, k, s>\frac{7}{10}, k+s>\frac{3}{2}$. Moreover, in each of these cases, a polynomial in time bound is obtained for the growth of the norms. The method of almost conservation laws has also been applied in [1] to obtain global solutions for a wave-Schrödinger system in dimensions 3 and 4 that in particular, for $d=3$, implies GWP for (1.4) with $k=s, s>\frac{\sqrt{57}-5}{4}$. In this paper, we prove GWP for $d=3, k=s=0$, by a scheme involving (1.5) and direct application of the Strichartz estimates for the Schrödinger operator and Minkowski's integral inequality applied to the Klein-Gordon Duhamel term 3

Theorem 1.3. If $\alpha \beta>0$, the Klein-Gordon-Schrödinger system (1.4) in dimension $d=3$ is globally well-posed for $\left(u_{0}, n_{0}, n_{1}\right) \in L^{2} \times L^{2} \times H^{-1}$. Moreover, the solution $(u, n)$ satisfies (1.5) and

$$
\|n(t)\|_{L^{2}}+\left\|\partial_{t} n(t)\right\|_{H^{-1}} \leq \exp \left(c|t|\left\|u_{0}\right\|_{L^{2}}^{2}\right) \max \left(\left(\left\|n_{0}\right\|_{L^{2}}+\left\|n_{1}\right\|_{H^{-1}}\right),\left\|u_{0}\right\|_{L^{2}}^{2}\right) .
$$

Remark 1.4. In the case where $\alpha<0$ and $\beta<0$, global well-posedness of (1.4) for large smooth data was previously unknown. Since our proof of Theorem 1.3 is based on the conservation of $\|u(t)\|_{L^{2}}$, we do not require any Sobolev norm control obtained from the Hamiltonian, and we obtain global well-posedness for this case as well.

The proof of both Theorem 1.1 and 1.3 apply essentially the same scheme, although they invoke a different space-time norm in the local theory estimates.

1.1. Outline of method. We describe the globalization scheme for the Zakharov system and the Klein-Gordon-Schrödinger system using the abstract initial value problem posed at some time $t=T_{j}$ :

$$
\left\{\begin{array}{l}
K u=F(u, n), \\
L n=G(u), \\
(u, n)\left(T_{j}\right)=\left(u_{j}, n_{j}\right) .
\end{array}\right.
$$

\footnotetext{
${ }^{3}$ Similar results hold for $d=1, d=2$, although for expositional convenience, we have restricted ourselves to the most delicate case, $d=3$.
} 
Here $K$ and $L$ are linear differential operators of evolution type, $F$ is a nonlinear term coupling the two equations together and $G$ is a nonlinear term depending only upon $u$. The fact that $G$ does not depend upon $n$ is used in our scheme. Let $W(t) n_{0}$ denote the linear group $W(t)$ applied to initial data $n_{0}$ solving the initial value problem $L n=0, n(0)=n_{0}$. Similarly, let $S(t) u_{0}$ denote the solution of $K u=0, u(0)=u_{0}$. We denote with $W n_{0}+L^{-1} g$ the solution of the linear initial value problem $L u=g, n(0)=n_{0}$. Similarly, $S u_{0}+K^{-1} g$ denotes the solution of $K u=g, u(0)=u_{0}$.

We solve the second equation in our system to define $n$ in terms of the initial data $n_{j}$ and $u$,

$$
n=W n_{j}+L^{-1} G(u),
$$

and insert the result into the solution formula for $u$ to obtain an integrodifferential equation for $u$,

$$
u=S u_{j}+K^{-1} F\left(u, W n_{j}+L^{-1} G(u)\right) .
$$

Local well-posedness for problems of the form (1.8) often follows from a fixed point argument applied to (1.10). The fixed point analysis is carried out in a Banach space $X_{\left[T_{j}, T_{j-1}\right]}$ of functions defined on the spacetime slab $\left[T_{j}, T_{j+1}\right] \times \mathbb{R}^{d}$. The initial data are considered in function spaces having the unitarity property with respect to the linear solution maps

$$
\left\|W(t) n_{0}\right\|_{\mathcal{W}}=\left\|n_{0}\right\|_{\mathcal{W}},\left\|S(t) u_{0}\right\|_{\mathcal{S}}=\left\|u_{0}\right\|_{\mathcal{S}}, \forall t
$$

and $X_{\left[T_{j}, T_{j+1}\right]} \subset C\left(\left[T_{j}, T_{j+1}\right] ; \mathcal{S}\right)$. For the applications we have in mind, the length of the time interval $\Delta_{j}:=\left|\left[T_{j}, T_{j+1}\right]\right|$ is chosen to be small enough to prove a contraction estimate and the smallness condition is of the form

$$
\Delta_{j} \leq \min \left(\left\|u_{j}\right\|_{\mathcal{S}}^{-\gamma},\left\|n_{j}\right\|_{\mathcal{W}}^{-\beta}\right)
$$

for certain $\gamma, \beta>0$.

Suppose that $\|u(t)\|_{\mathcal{S}}=\left\|u_{0}\right\|_{\mathcal{S}}$ for all times $t$ where solutions of (1.8) are well defined. If we iterate the local well-posedness argument, we will have successive time intervals $\left[T_{j}, T_{j+1}\right]$ with uniformly lower bounded lengths unless $\left\|n_{j}\right\|_{\mathcal{W}}$ grows without bound as we increase $j$. Suppose then at some time $T_{j}$ we have $\left\|n_{j}\right\|_{\mathcal{W}} \gg$ $\left\|u_{j}\right\|_{\mathcal{S}}^{\gamma / \beta}$ so that $\Delta_{j}=\left\|n_{j}\right\|_{\mathcal{W}}^{-\beta}$.

Since we have that (1.9) and (1.11) hold, any growth in $\|n(t)\|_{\mathcal{W}}$ as $t$ moves through the time interval $\left[T_{j}, T_{j+1}\right]$ is due to the nonlinear influence of $u$ upon $n$ through the term $L^{-1} G(u)$. Therefore, an estimate of the form

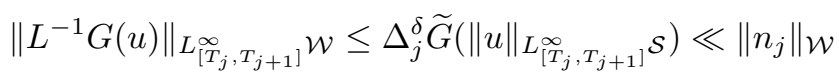

permits an iteration of the local theory. Observe that the appearance of the conserved $\mathcal{S}$ norm of $u$ in this step suggests that we should retain this smallness property of the $\mathcal{W}$ increment of $n$ over $\left[T_{j}, T_{j+1}\right]$ uniformly with respect to $j$. We then iterate the local well-posedness argument

$$
m=O\left(\frac{\left\|n_{j}\right\|_{\mathcal{W}}}{\Delta^{\delta} \widetilde{G}\left(\left\|u_{0}\right\|_{\mathcal{S}}\right)}\right)
$$


times with time steps of uniform size $\Delta=\left(2\left\|n_{j}\right\|_{\mathcal{W}}\right)^{-\beta}$. This extends the solution to the time interval $\left[T_{j}, T_{j}+m \Delta\right]$ with

$$
m \Delta=C\left(\left\|u_{0}\right\|_{\mathcal{S}}\right)\left\|n_{j}\right\|_{\mathcal{W}}^{1-\beta+\delta \beta} .
$$

If $1-\beta+\delta \beta \geq 0$, the scheme progresses to give global well-posedness for (1.8).

Implementing this abstract scheme for specific systems requires a quantification of the parameters $\beta$ and $\delta$ using the local-in-time theory for the system. Notice that one way to force $1-\beta+\delta \beta \geq 0$ is by demanding $\beta \leq 1$. But $\beta$ is always bigger than 1. Still $1-\beta+\delta \beta \geq 0$ can be greater than or equal to zero because of the contribution of the $\delta \beta$ term for certain $\beta>1$ and $\delta>0$. Calculations are required to obtain the parameters $\beta, \delta$ in any particular system. Unfortunately, we will often find that $\delta$ is very close to zero. Thus the condition that $1-\beta-\delta \beta \geq 0$ fails to hold for many physical systems. Nevertheless for the Klein-Gordon-Schrödinger system the local well-posedness theory that we develop using Strichartz's norms is sufficient for the above condition to hold. In particular we have that $\beta=4$ and $\delta=\frac{3}{4}$ (see the proof of Theorem 1.3) and thus $1-\beta+\delta \beta=0$. This approach cannot be used for the Zakharov system. The main reason is that the nonlinearity $G(u)$ has two derivatives (see equation (1.1)) and the local estimates are not as generous. The idea now is to perform the contraction argument for (1.10) in a ball

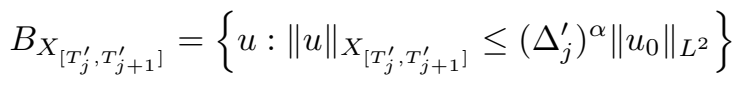

where $\Delta_{j}^{\prime}=\left|\left[T_{j}^{\prime}, T_{j+1}^{\prime}\right]\right|$. This idea is implemented here through the use of the $X^{s, b}$ spaces with $b<1 / 2$. An easy consequence of this new iteration is that the local time interval $\Delta_{j}^{\prime}$ is larger, or in other words (since $\Delta^{\prime}<1$ ) $\beta$ is smaller. In addition when we calculate the growth of the $\|n(t)\|_{\mathcal{W}}$ norm, it takes more time for this norm to double in size. In other words the new $\delta^{\prime}$ is bigger. Thus since $m^{\prime}>m$ and $\Delta^{\prime}>\Delta$ we have a better chance to meet the requirement of

$$
m \Delta \gtrsim 1 \Longleftrightarrow 1-\beta+\delta \beta \geq 0 .
$$

The details are explained in the proof of Theorem 1.1

Remark 1.5. The abstract scheme described above can be applied to other evolution systems that have common features with systems (1.1) and (1.4). In particular, the scheme requires a satisfactory local well-posedness theory in a Banach space that embeds in $C\left(\left[T_{j}, T_{j+1}\right] ; \mathcal{S}\right)$, with $\|u(t)\|_{\mathcal{S}}=\left\|u_{0}\right\|_{\mathcal{S}}$ holding true, and that the nonlinear term of the second equation is independent of $n$. As examples we mention the following systems.

The initial-value problem for the coupled Schrödinger-Airy equation is

$$
\left\{\begin{array}{l}
i \partial_{t} u+\partial_{x}^{2} u=\alpha u n+\beta|u|^{2} u, \quad x \in \mathbb{R}, t \in \mathbb{R}, \\
\partial_{t} n+\partial_{x}^{3} n=\gamma \partial_{x}|u|^{2} \\
u(x, 0)=u_{0}(x), n(x, 0)=n_{0}(x) .
\end{array}\right.
$$

This system arises in the theory of capillary-gravity waves. The local well-posedness theory has been successively sharpened in [2], 3], 9], the last paper establishing local well-posedness for $\left(u_{0}, n_{0}\right) \in L^{2} \times H^{s}$ for $-\frac{3}{4}<s \leq-\frac{1}{2}$, and in some more regular spaces. In [20, Pecher proved global well-posedness using $I$-method techniques for the harder Schrödinger-KdV system where the left hand side of the second equation of (1.13) includes $n \partial_{x} n$, with $\left(u_{0}, n_{0}\right) \in H^{s} \times H^{s}$ and $s>\frac{3}{5}$ when $\beta=0$, and 
also for $s>\frac{2}{3}$ when $\beta \neq 0$ by dropping down from the $s=1$ setting in which conservation of energy yields global well-posedness.

Our scheme also applies to the Schrödinger-Benjamin-Ono system,

$$
\left\{\begin{array}{l}
i \partial_{t} u+\partial_{x}^{2} u=\alpha u n, \\
\partial_{t} n+\nu \partial_{x}\left|\partial_{x}\right| n=\beta \partial_{x}|u|^{2}, \\
u(x, 0)=u_{0}(x), n(x, 0)=n_{0}(x)
\end{array} \quad x \in \mathbb{R}, t \in \mathbb{R}\right.
$$

with $\alpha, \beta, \nu \in \mathbb{R}$. This system has been studied in 3] where local well-posedness for $u_{0} \in H^{s}$ and $n_{0} \in H^{s-\frac{1}{2}}$, with $s \geq 0$ and $|\nu| \neq 1$, is established. In particular it is locally well-posed for $\left(u_{0}, n_{0}\right) \in L^{2} \times H^{-1 / 2}$. Pecher proved [19] global wellposedness for $s>1 / 3$ under the parameter constraints $\nu>0, \frac{\alpha}{\beta}<0$ and also proved local well-posedness without the restriction $|\nu| \neq 1$ but only for $s>0$. In a forthcoming paper, we establish global well-posedness results for (1.13) and (1.14) with $|\nu| \neq 1$ for $\left(u_{0}, n_{0}\right) \in L^{2} \times H^{-1 / 2}$.

\section{Basic estimates for the Group And Duhamel terms}

Let $U(t)=e^{i t \Delta}$ denote the free linear Schrödinger group. For the $1 \mathrm{~d}$ wave equation, it is convenient to factor the wave operator $\partial_{t}^{2}-\partial_{x}^{2}=\left(\partial_{t}-\partial_{x}\right)\left(\partial_{t}+\partial_{x}\right)$, and work with "reduced" components, as was done in [11]. Low frequencies in the time-derivative initial data create some minor difficulties, which we address in a manner slightly different than was done in 11. Consider an initial data pair $\left(n_{0}, n_{1}\right)$, and we look to solve $\left(\partial_{t}^{2}-\partial_{x}^{2}\right) n=0$ such that $n(0)=n_{0}, \partial_{t} n(0)=n_{1}$. Split $n_{1}=n_{1 L}+n_{1 H}$ into low and high frequencies, and set $\hat{\nu}(\xi)=\frac{\hat{n}_{1 H}(\xi)}{i \xi}$, so that $\partial_{x} \nu=n_{1 H}$. Let

$$
\begin{aligned}
& W_{+}\left(n_{0}, n_{1}\right)(t, x)=\frac{1}{2} n_{0}(x-t)-\frac{1}{2} \nu(x-t)+\frac{1}{2} \int_{x-t}^{x} n_{1 L}(y) d y \\
& W_{-}\left(n_{0}, n_{1}\right)(t, x)=\frac{1}{2} n_{0}(x+t)+\frac{1}{2} \nu(x+t)+\frac{1}{2} \int_{x}^{x+t} n_{1 L}(y) d y
\end{aligned}
$$

so that

$$
\begin{gathered}
\left(\partial_{t} \pm \partial_{x}\right) W_{ \pm}\left(n_{0}, n_{1}\right)(t, x)=\frac{1}{2} n_{1 L}(x) \\
W_{ \pm}\left(n_{0}, n_{1}\right)(x, 0)=\frac{1}{2} n_{0}(x) \mp \frac{1}{2} \nu(x) \\
\partial_{t} W_{ \pm}\left(n_{0}, n_{1}\right)(x, 0)=\mp \frac{1}{2} \partial_{x} n_{0}(x)+\frac{1}{2} \partial_{x} \nu(x)+\frac{1}{2} n_{1 L}(x),
\end{gathered}
$$

and thus $n=W_{+}\left(n_{0}, n_{1}\right)+W_{-}\left(n_{0}, n_{1}\right)$ has the desired properties. We shall also use the notation $W\left(n_{0}, n_{1}\right)=W_{+}\left(n_{0}, n_{1}\right)+W_{-}\left(n_{0}, n_{1}\right)$. Let

$$
G(t)\left(n_{0}, n_{1}\right)=\cos \left[t(I-\Delta)^{1 / 2}\right] n_{0}+\frac{\sin \left[t(I-\Delta)^{1 / 2}\right]}{(I-\Delta)^{1 / 2}} n_{1}
$$

be the free linear Klein-Gordon group, so that $\left(\partial_{t}^{2}+(1-\Delta)\right) G(t)\left(n_{0}, n_{1}\right)=0$, $G(0)\left(n_{0}, n_{1}\right)=n_{0}, \partial_{t} G(0)\left(n_{0}, n_{1}\right)=n_{1}$. Since our analysis involves tracking quantities whose size increments, rather than doubles, from one step to the next, it is imperative that we be precise about the definition of the following Sobolev norms. When we write the norm $H^{s}$, we shall mean exactly

$$
\|f\|_{H^{s}}=\left(\int_{\xi}\left(1+|\xi|^{2}\right)^{s}|\hat{f}(\xi)|^{2} d \xi\right)^{1 / 2} .
$$


Define the norm

$$
\|f\|_{A^{s}}=\left(\int_{|\xi| \leq 1}|\hat{f}(\xi)|^{2} d \xi+\int_{|\xi| \geq 1}|\xi|^{2 s}|\hat{f}(\xi)|^{2} d \xi\right)^{1 / 2} .
$$

Of course, $\|f\|_{A^{s}} \sim\|f\|_{H^{s}}$. Let

$$
\left\|\left(n_{0}, n_{1}\right)\right\| \mathcal{W}=\left(\left\|n_{0}\right\|_{A_{x}^{-1 / 2}}^{2}+\left\|n_{1}\right\|_{A_{x}^{-3 / 2}}^{2}\right)^{1 / 2}
$$

When working with a function of $t$, we use the shorthand

$$
\|n(t)\|_{\mathcal{W}}=\left\|\left(n(t), \partial_{t} n(t)\right)\right\|_{\mathcal{W}} .
$$

In our treatment of the Zakharov system, we shall track the size of the wave component $n(t)$ in the above norm. Let

$$
\left\|\left(n_{0}, n_{1}\right)\right\|_{\mathcal{G}}=\left(\left\|n_{0}\right\|_{L_{x}^{2}}^{2}+\left\|n_{1}\right\|_{H_{x}^{-1}}^{2}\right)^{1 / 2} .
$$

Again, for functions of $t$, we use the shorthand $\|n(t)\|_{\mathcal{G}}=\left\|\left(n(t), \partial_{t} n(t)\right)\right\|_{\mathcal{G}}$. In our treatment of the Klein-Gordon-Schrödinger system, we shall track the size of the wave component $n(t)$ in the above norm.

In our treatment of the Zakharov system, we shall need to work in the Bourgain spaces. We define the Schrödinger-Bourgain space $X_{0, \alpha}^{S}, \alpha \in \mathbb{R}$, by the norm

$$
\|z\|_{X_{0, \alpha}^{S}}=\left(\iint_{\xi, \tau}\left\langle\tau+|\xi|^{2}\right\rangle^{2 \alpha}|\hat{z}(\xi, \tau)|^{2} d \xi d \tau\right)^{1 / 2},
$$

and the one-dimensional reduced-wave-Bourgain spaces $X_{-\frac{1}{2}, \alpha}^{W \pm}$, for $\alpha \in \mathbb{R}$, as

$$
\|z\|_{X_{-\frac{1}{2}, \alpha}^{W_{ \pm}}}=\left(\iint_{\xi, \tau}\langle\xi\rangle^{-1}\langle\tau \pm \xi\rangle^{2 \alpha}|\hat{z}(\xi, \tau)|^{2} d \xi d \tau\right)^{1 / 2} .
$$

Let $\psi \in C_{0}^{\infty}(\mathbb{R})$ satisfy $\psi(t)=1$ on $[-1,1]$ and $\psi(t)=0$ outside of $[-2,2]$. Let $\psi_{T}(t)=\psi(t / T)$, which will serve as a time cutoff for the Bourgain space estimates. For clarity, we write $\psi_{1}(t)=\psi(t)$. The following two lemmas are standard in the subject, although we are focusing our attention particularly on the exponent of $T$ in these estimates.

Lemma 2.1 (Group estimates). Suppose $T \leq 1$.

(a) Schrödinger. $\left\|U(t) u_{0}\right\|_{C\left(\mathbb{R}_{t} ; L_{x}^{2}\right)}=\left\|u_{0}\right\|_{L_{x}^{2}}$.

If $0 \leq b_{1} \leq \frac{1}{2}$, then $\left\|\psi_{T}(t) U(t) u_{0}\right\|_{X_{0, b_{1}}^{S}} \lesssim T^{\frac{1}{2}-b_{1}}\left\|u_{0}\right\|_{L^{2}}$.

(Strichartz estimates). If $2 \leq q \leq \infty, 2 \leq r \leq \infty, \frac{2}{q}+\frac{d}{r}=\frac{d}{2}$, excluding the case $d=2, q=2, r=\infty$, then $\left\|U(t) u_{0}\right\|_{L_{t}^{q} L_{r}^{r}} \lesssim\left\|u_{0}\right\|_{L^{2}}$.

(b) 1-d Wave. $\left\|W(t)\left(n_{0}, n_{1}\right)\right\|_{C\left([0, T] ; \mathcal{W}_{x}\right)} \leq(1+T)\left\|\left(n_{0}, n_{1}\right)\right\|_{\mathcal{W}}$.

$$
\text { If } 0 \leq b \leq \frac{1}{2},\left\|\psi_{T}(t) W_{ \pm}(t)\left(n_{0}, n_{1}\right)\right\|_{X_{-\frac{1}{2}, b}^{W_{ \pm}}} \lesssim T^{\frac{1}{2}-b}\left\|\left(n_{0}, n_{1}\right)\right\|_{\mathcal{W}} .
$$

(c) Klein-Gordon. $\left\|G(t)\left(n_{0}, n_{1}\right)\right\|_{C\left(\mathbb{R}_{t} ; \mathcal{G}_{x}\right)}=\left\|\left(n_{0}, n_{1}\right)\right\|_{\mathcal{G}}$.

Remark 2.2. It is important that the first estimate in (B) and the identity in (IC) do not have implicit constant multiples on the right-hand side, as these estimates will be used to deduce almost conservation laws. The $(1+T)$ prefactor in the first estimate of (b) arises from the low frequency terms. Had we made the assumption that $n_{1} \in \dot{H}^{-1}$, this term could be removed and the norm $\mathcal{W}$ redefined so that 
equality is obtained. The $(1+T)$ prefactor will not cause trouble in our iteration since $T$ will be selected so that $T\left\|\left(n_{0}, n_{1}\right)\right\|_{\mathcal{W}}$ functions as an increment whose size is on par with the increment arising from the Duhamel terms (see the proof of Theorem 1.1 for details).

Proof. The Strichartz estimates quoted in (国) were established in 21] (for a more recent reference, see [13]). The first assertion in (国) is immediate by Plancherel's theorem. For the second assertion in (国), we note that $\left[\psi_{T}(t) U(t) u_{0}\right] \mathcal{Y}(\xi, \tau)=$ $\left.\left(\psi_{T}\right) \mathcal{( \tau}+\xi^{2}\right) \hat{u}_{0}(\xi)$, and consequently

$$
\left\|\psi_{T}(t) U(t) u_{0}\right\|_{X_{0, b_{1}}^{S}} \leq c\left\|\psi_{T}\right\|_{H^{b_{1}}}\left\|u_{0}\right\|_{L^{2}} .
$$

To complete the proof of the estimate, we note that

$$
\left\|\psi_{T}\right\|_{H^{b_{1}}} \leq\left\|\psi_{T}\right\|_{L^{2}}+\left\|\psi_{T}\right\|_{\dot{H}^{b_{1}}}=T^{\frac{1}{2}}\left\|\psi_{1}\right\|_{L^{2}}+T^{\frac{1}{2}-b_{1}}\left\|\psi_{1}\right\|_{\dot{H}^{b_{1}}}
$$

by scaling.

For the first assertion in (b), let $f(x, t)$ solve the linear wave equation

$$
\partial_{t}^{2} f-\partial_{x}^{2} f=0
$$

with initial data $f(x, 0)=n_{0}(x), \partial_{t} f(x, 0)=n_{1}(x)$. Let $P_{H}$ be the projection onto frequencies $|\xi| \geq 1$, and $P_{L}$ be the projection onto frequencies $|\xi| \leq 1$. Let $D^{-3 / 2}$ be the multiplier operator with symbol $|\xi|^{-3 / 2}$. By applying $D^{-3 / 2} P_{H}$ to (2.4), multiplying by $D^{-3 / 2} P_{H} \partial_{t} f$ and integrating in $x$, we obtain the conservation identity

$$
\left\|P_{H} f(T)\right\|_{A_{x}^{-1 / 2}}^{2}+\left\|\partial_{t} P_{H} f(T)\right\|_{A_{x}^{-3 / 2}}^{2}=\left\|P_{H} n_{0}\right\|_{A^{-1 / 2}}^{2}+\left\|P_{H} n_{1}\right\|_{A^{-3 / 2}}^{2}
$$

To obtain low frequency estimates, we work directly from the explicit formula

$$
f(x, t)=\frac{1}{2} n_{0}(x+t)+\frac{1}{2} n_{0}(x-t)+\frac{1}{2} \int_{x-t}^{x+t} n_{1}(y) d y .
$$

By applying $P_{L}$ and then directly estimating, we obtain

$$
\left\|P_{L} f(T)\right\|_{L_{x}^{2}} \leq\left\|P_{L} n_{0}\right\|_{L^{2}}+T\left\|P_{L} n_{1}\right\|_{L^{2}} .
$$

After applying $\partial_{t}$ to (2.6), it can be rewritten as

$$
\partial_{t} f(x, t)=\frac{1}{2} \int_{x-t}^{x+t} \partial^{2} n_{0}(y) d y+\frac{1}{2} n_{1}(x+t)+\frac{1}{2} n_{1}(x-t) .
$$

Applying $P_{L}$ and then directly estimating, we obtain

$$
\left\|P_{L} \partial_{t} f(T)\right\|_{L_{x}^{2}} \leq T\left\|P_{L} n_{0}\right\|_{L^{2}}+\left\|P_{L} n_{1}\right\|_{L^{2}} .
$$

Combining (2.5), (2.7), and (2.8), we obtain the claim.

The second part of (b) is proved similarly to the second part of (国).

For (ㄷ), let $f(t, x)$ solve

$$
\partial_{t}^{2} f-\Delta f+f=0
$$

with initial data $\left(f(0), \partial_{t} f(0)\right)=\left(n_{0}, n_{1}\right)$. Let $E$ be the multiplier operator with symbol $\left(1+|\xi|^{2}\right)^{-1 / 2}$. Apply $E$ to (2.9), then multiply by $\partial_{t} E f$, and finally integrate in $x$ to obtain the asserted conservation law. 
Let

$$
U *_{R} z(t, x)=\int_{0}^{t} U\left(t-t^{\prime}\right) z\left(t^{\prime}, x\right) d t^{\prime}
$$

denote the Duhamel operator corresponding to the Schrödinger operator, so that $\left(i \partial_{t}+\Delta\right) U *_{R} z(t, x)=i z(t, x), U *_{R} z(0, x)=0$. Let

$$
W_{ \pm} *_{R} z(t, x)=\frac{1}{2} \int_{0}^{t} z(t-s, x \mp s) d s
$$

so that

$$
\begin{gathered}
\left(\partial_{t} \pm \partial_{x}\right) W_{ \pm} *_{R} z(t, x)=\frac{1}{2} z(t, x), \\
W_{ \pm} *_{R} z(0, x)=0, \quad \partial_{t} W_{ \pm} *_{R} z(0, x)=\frac{1}{2} z(0, x) .
\end{gathered}
$$

It follows that if we set $n=W_{+} *_{R} z-W_{-} *_{R} z$, then $\left(\partial_{t}^{2}-\partial_{x}^{2}\right) n=\partial_{x} z$ and $n(0, x)=0, \partial_{t} n(0, x)=0$, so we define

$$
W *_{R} z=W_{+} *_{R} z-W_{-} *_{R} z .
$$

For the Klein-Gordon equation, let

$$
G *_{R} z(t, x)=\int_{0}^{t} \frac{\sin \left[\left(t-t^{\prime}\right)(I-\Delta)^{1 / 2}\right]}{(I-\Delta)^{1 / 2}} z\left(t^{\prime}, x\right) d t^{\prime}
$$

so that $\left(\partial_{t}^{2}+(I-\Delta)\right) G *_{R} z=z, G *_{R} z(0, x)=0, \partial_{t} G *_{R} z(0, x)=0$.

Lemma 2.3 (Duhamel estimates). Suppose $T \leq 1$.

(a) Schrödinger. If $0 \leq c_{1}<\frac{1}{2}$, then $\left\|U *_{R} z\right\|_{C\left([0, T] ; L_{x}^{2}\right)} \lesssim T^{\frac{1}{2}-c_{1}}\|z\|_{X_{0,-c_{1}}^{S}}$. If $0 \leq c_{1}<\frac{1}{2}, 0 \leq b_{1}, b_{1}+c_{1} \leq 1$, then $\left\|\psi_{T} U *_{R} z\right\|_{X_{0, b_{1}}^{S}} \lesssim T^{1-b_{1}-c_{1}}\|z\|_{X_{0,-c_{1}}^{S}}$. (Strichartz estimates). If $2 \leq q \leq \infty, 2 \leq r \leq \infty, \frac{2}{q}+\frac{d}{r}=\frac{d}{2}$ excluding the case $d=2, q=2, r=\infty$, and similarly for $\tilde{q}, \tilde{r}$, then $\left\|U *_{R} z\right\|_{C\left([0, T] ; L_{x}^{2}\right)}+$ $\left\|U *_{R} z\right\|_{L_{[0, T]}^{q} L_{x}^{r}} \lesssim\|z\|_{L_{[0, T]}^{\tilde{q}^{\prime}} L_{x}^{\tilde{r}^{\prime}}}$, where' indicates the Hölder dual exponent $\left(\frac{1}{p}+\frac{1}{p^{\prime}}=1\right)$.

(b) 1-d Wave. If $0 \leq c<\frac{1}{2}$, then $\left\|W *_{R} z\right\|_{C\left([0, T] ; \mathcal{W}_{x}\right)} \lesssim T^{\frac{1}{2}-c}\|z\|_{X_{-\frac{1}{2},-c}^{W \pm}}$.

$$
\text { If } 0 \leq c<\frac{1}{2}, 0 \leq b, b+c \leq 1 \text {, then }\left\|\psi_{T} W_{ \pm} *_{R} z\right\|_{X_{-\frac{1}{2}, b}^{W \pm}} \lesssim T^{1-b-c}\|z\|_{X_{-\frac{1}{2},-c}^{W \pm}} .
$$

(c) Klein-Gordon. $\left\|G *_{R} z\right\|_{C\left([0, T] ; \mathcal{G}_{x}\right)} \lesssim\|z\|_{L_{[0, T]}^{1} H_{x}^{-1}}$.

Proof. The second assertion in each of (国) and (B) is [11, Lemma 2.1(ii). For the Stricharz estimates quoted in (国), see [21], [13]. We next establish the first part of (国). We begin by establishing the bound

$$
\left\|\psi_{T}(t) U *_{R} z(x, t)\right\|_{L_{t}^{\infty} L_{x}^{2}} \leq c T^{\frac{1}{2}-c_{1}}\|z\|_{X_{0,-c_{1}}^{S}} .
$$

Let $f_{\xi}(t)=e^{i t \xi^{2}} \hat{z}(\xi, t)$, where ^ denotes the Fourier transform in the $x$-variable only. We have

$$
\begin{aligned}
& \left\|\psi_{T}(t) U *_{R} z(x, t)\right\|_{L_{t}^{\infty} L_{x}^{2}} \\
& =\left\|\psi_{T}(t) \int_{0}^{t} f_{\xi}\left(t^{\prime}\right) d t^{\prime}\right\|_{L_{t}^{\infty} L_{\xi}^{2}} \\
& \leq\left\|\psi_{T}(t) \int_{0}^{t} f_{\xi}\left(t^{\prime}\right) d t^{\prime}\right\|_{L_{\xi}^{2} L_{t}^{\infty}} .
\end{aligned}
$$


Below we shall show that for a function $f(t)$ of the $t$-variable alone, we have the estimate

$$
\left\|\psi_{T}(t) \int_{0}^{t} f\left(t^{\prime}\right) d t^{\prime}\right\|_{L_{t}^{\infty}} \leq c T^{\frac{1}{2}-c_{1}}\|f\|_{H^{-c_{1}}}
$$

Assuming this, it then follows from (2.12) that

$$
\left\|\psi_{T}(t) U *_{R} z(x, t)\right\|_{L_{t}^{\infty} L_{x}^{2}} \leq c T^{\frac{1}{2}-c_{1}}\|\| f_{\xi}\left\|_{H_{t}^{-c_{1}}}\right\|_{L_{\xi}^{2}}=c T^{\frac{1}{2}-c_{1}}\|z\|_{X_{0,-c_{1}}^{S}},
$$

completing the proof of (2.11). Now we show (2.13). Break $f(t)=f_{+}(t)+f_{-}(t)$ where $\hat{f}_{-}(\tau)=\chi_{|\tau|<\frac{1}{T}} \hat{f}(\tau)$ and $\hat{f}_{+}(\tau)=\chi_{|\tau|>\frac{1}{T}} \hat{f}(\tau)$. Then for $f_{-}$, we have

$$
\left\|\psi_{T}(t) \int_{0}^{t} f_{-}(s) d s\right\|_{L_{t}^{\infty}} \leq T^{1 / 2}\left\|f_{-}\right\|_{L^{2}} \leq T^{\frac{1}{2}-c_{1}}\left\|f_{-}\right\|_{H^{-c_{1}}} .
$$

We compute

$$
\begin{array}{r}
\int_{0}^{t} f_{+}(s) d s=\left(\chi_{[-t, 0]} * f_{+}\right)(0) \\
\quad=\frac{1}{2 \pi} \int\left(\chi_{[-t, 0]} * f_{+}\right)(\sigma) d \sigma \\
\quad=\frac{1}{2 \pi} \int_{\sigma} \frac{1-e^{-i t \sigma}}{i \sigma} \hat{f}_{+}(\sigma) d \sigma
\end{array}
$$

and hence

$$
\left[\psi_{T}(t) \int_{0}^{t} f_{+}(s) d s\right] \hat{(\tau)}=\int_{\sigma} \frac{T \hat{\psi}(T(\tau-\sigma))-T \hat{\psi}(T \tau)}{\sigma} \hat{f}_{+}(\sigma) d \sigma
$$

Thus,

$$
\begin{aligned}
& \left\|\psi_{T}(t) \int_{0}^{t} f_{+}(s) d s\right\|_{L_{t}^{\infty}} \\
& \quad \leq\|T \hat{\psi}(T \tau)\|_{L_{\tau}^{1}}\left\|\frac{\hat{f}_{+}(\tau)}{\tau}\right\|_{L_{\tau}^{1}} \\
& \quad \leq\left(\int_{|\tau|>\frac{1}{T}} \frac{d \tau}{|\tau|^{2-2 c_{1}}}\right)^{1 / 2}\|f\|_{H^{-c_{1}}} \\
& \leq T^{\frac{1}{2}-c_{1}}\|f\|_{H^{-c_{1}}},
\end{aligned}
$$

establishing (2.13). It remains only to show continuity, i.e. that for a fixed $z \in$ $X_{0,-c_{1}}^{S}$ and each $\epsilon>0$, there is $\delta=\delta(\epsilon, z)>0$ such that if $\left|t_{2}-t_{1}\right|<\delta$, then

$$
\left\|\psi_{T} U *_{R} z\left(x, t_{2}\right)-\psi_{T} U *_{R} z\left(x, t_{1}\right)\right\|_{L_{x}^{2}}<\epsilon .
$$

By an $\epsilon / 3$ argument appealing to (2.11), it suffices to establish this statement for $z$ belonging to the dense class $\mathcal{S}\left(\mathbb{R}^{2}\right) \subset X_{0,-c_{1}}^{S}$. However, if $z \in \mathcal{S}\left(\mathbb{R}^{2}\right)$, we have $\partial_{t}\left(U *_{R} z\right)=z+i \Delta\left(U *_{R} z\right)$, and the fundamental theorem of calculus and (2.11) imply that

$$
\left\|U *_{R} z\left(\cdot, t_{2}\right)-U *_{R} z\left(\cdot, t_{1}\right)\right\|_{L_{x}^{2}} \leq c\left(t_{2}-t_{1}\right)\left(\|z\|_{L_{t}^{\infty} L_{x}^{2}}+\|\Delta z\|_{X_{0,-c_{1}}^{S}}\right) .
$$


The proof of the first assertion of (b) proceeds in analogy to the above proof, first establishing the bound

$$
\left\|W_{ \pm} *_{R} z\right\|_{L_{t}^{\infty} H_{x}^{-1 / 2}} \lesssim T^{\frac{1}{2}-c}\|z\|_{X_{-\frac{1}{2},-c}^{W \pm}} .
$$

The continuity statement is deduced by a density argument as in the previous paragraph, and finally the bound as stated on $W *_{R} z$ follows by the identity

$$
\partial_{t} W *_{R} z=\partial_{x} W_{+} *_{R} z+\partial_{x} W_{-} *_{R} z .
$$

The proof of (ㄷ) follows from an application of Minkowskii's integral inequality, with the continuity statement deduced by a density argument as in the previous paragraph.

\section{1-D ZAKHAROV SYSTEM}

In this section, we prove Theorem 1.1. We shall make use of the conservation law (1.2) to control the growth of $u(t)$ from one local time step to the next. We track the growth of $n(t)$ in the norm $\mathcal{W}$ defined in (2.2) using the estimates from the local theory. We now state the needed estimates from the local theory of [1].

Lemma 3.1 (Multilinear estimates).

(a) If $\frac{1}{4}<b_{1}, c_{1}, b<\frac{1}{2}$ and $b+b_{1}+c_{1} \geq 1$, then

$$
\left\|n_{ \pm} u\right\|_{X_{0,-c_{1}}^{S}} \lesssim\left\|n_{ \pm}\right\|_{X_{-\frac{1}{2}, b}^{W \pm}}\|u\|_{X_{0, b_{1}}^{S}} .
$$

(b) If $\frac{1}{4}<b_{1}, c<\frac{1}{2}$ and $2 b_{1}+c \geq 1$, then

$$
\left\|\partial_{x}\left(u_{1} \bar{u}_{2}\right)\right\|_{X_{-\frac{1}{2},-c}^{W \pm}} \lesssim\left\|u_{1}\right\|_{X_{0, b_{1}}^{S}}\left\|u_{2}\right\|_{X_{0, b_{1}}^{S}} .
$$

We remark that we can simultaneously achieve both optimal conditions $b+b_{1}+c_{1}=1$ and $2 b_{1}+c=1$, for example by taking all four indices $b=b_{1}=c=c_{1}=\frac{1}{3}$.

Proof. (回) is the case $k=0, \ell=-\frac{1}{2}$ in [11, Lemma 4.3, and (b) is the case $k=0$, $l=-\frac{1}{2}$ in [11, Lemma 4.4. The assumptions $b+c_{1}>\frac{3}{4}, b+b_{1}>\frac{3}{4}$ for Lemma 4.3 and $b_{1}+c>\frac{3}{4}$ for Lemma 4.4 that appear in [11] are not needed, and we only have the requirements $b+b_{1}+c_{1} \geq 1$ for Lemma 4.3 and $2 b_{1}+c \geq 1$ for Lemma 4.4. The reason is that equation (4.30) in [11] on p. 424 is finite even if $\alpha_{1}<\frac{1}{2}$ since the range of integration is finit 4 (from 0 to $\xi_{1}^{2} / 4$ ). Because relaxing this condition is essential to our method, we have included these proofs in the appendix so that they can be examined by the reader.

Proof of Theorem 1.1. As discussed above, we can reduce the wave component $n=$ $n_{+}+n_{-}$and recast (1.1) as

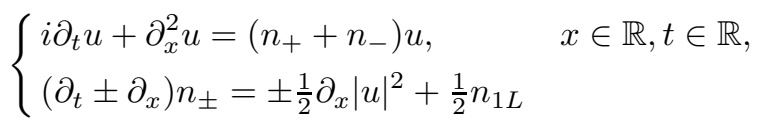

which has the integral equation formulation

$$
\begin{aligned}
u(t) & =U(t) u_{0}-i U *_{R}\left[\left(n_{+}+n_{-}\right) u\right](t), \\
n_{ \pm}(t) & =W_{ \pm}(t)\left(n_{0}, n_{1}\right) \pm W_{ \pm} *_{R}\left(\partial_{x}|u|^{2}\right)(t) .
\end{aligned}
$$

\footnotetext{
${ }^{4}$ This comment applies in the $k=0, \ell=-\frac{1}{2}$ setting, but perhaps not in the general setting in which Lemmas 4.3, 4.4 are stated.
} 
Fix $0<T<1$, and consider the maps $\Lambda_{S}, \Lambda_{W \pm}$ :

$$
\begin{aligned}
\Lambda_{S}\left(u, n_{ \pm}\right) & =\psi_{T} U u_{0}+\psi_{T} U *_{R}\left[\left(n_{+}+n_{-}\right) u\right], \\
\Lambda_{W \pm}(u) & =\psi_{T} W_{ \pm}\left(n_{0}, n_{1}\right) \pm \psi_{T} W_{ \pm} *_{R}\left(\partial_{x}|u|^{2}\right) .
\end{aligned}
$$

We seek a fixed point $\left(u(t), n_{ \pm}(t)\right)=\left(\Lambda_{S}\left(u, n_{ \pm}\right), \Lambda_{W \pm}(u)\right)$. Estimating (3.2) in $X_{0, b_{1}}^{S}$, applying the first estimates in Lemma 2.1(国), 2.3(国) and following through with Lemma 3.1(国); and estimating (3.3) in $X_{-\frac{1}{2}, b}^{W_{ \pm}}$, applying the first estimates in Lemma 2.1(b), 2.3(b) and following through with Lemma 3.1(b), we obtain

$$
\begin{aligned}
\left\|\Lambda_{S}\left(u, n_{ \pm}\right)\right\|_{X_{0, b_{1}}^{S}} & \lesssim T^{\frac{1}{2}-b_{1}}\left\|u_{0}\right\|_{L^{2}}+T^{1-b_{1}-c_{1}}\left\|n_{ \pm}\right\|_{X_{-\frac{1}{2}, b}^{W \pm}}\|u\|_{X_{0, b_{1}}^{S}}, \\
\left\|\Lambda_{W \pm}(u)\right\|_{X_{-\frac{1}{2}, b}^{W \pm}} & \lesssim T^{\frac{1}{2}-b}\left\|\left(n_{0}, n_{1}\right)\right\|_{\mathcal{W}}+T^{1-b-c}\|u\|_{X_{0, b_{1}}^{S}}^{2}
\end{aligned}
$$

and also

$$
\begin{aligned}
& \left\|\Lambda_{S}\left(u_{1}, n_{1 \pm}\right)-\Lambda_{S}\left(u_{2}, n_{2 \pm}\right)\right\|_{X_{0, b_{1}}^{S}} \\
& \quad \lesssim T^{1-b_{1}-c_{1}}\left(\left\|n_{1 \pm}\right\|_{X_{-\frac{1}{2}, b}^{W \pm}}\left\|u_{1}-u_{2}\right\|_{X_{0, b_{1}}^{S}}+\left\|n_{1 \pm}-n_{2 \pm}\right\|_{X_{-\frac{1}{2}, b}^{W \pm}}\left\|u_{2}\right\|_{X_{0, b_{1}}^{S}}\right), \\
& \left\|\Lambda_{W \pm}\left(u_{1}\right)-\Lambda_{W \pm}\left(u_{2}\right)\right\|_{X_{-\frac{1}{2}, b}^{W \pm}} \lesssim T^{1-b-c}\left(\left\|u_{1}\right\|_{X_{0, b_{1}}^{S}}+\left\|u_{2}\right\|_{X_{0, b_{1}}^{S}}\right)\left\|u_{1}-u_{2}\right\|_{X_{0, b_{1}}^{S}} .
\end{aligned}
$$

By taking $T$ such that

$$
\begin{gathered}
T^{\frac{3}{2}-2 b_{1}-c_{1}}\left\|u_{0}\right\|_{L^{2}} \lesssim 1, \quad T^{\frac{3}{2}-b-b_{1}-c}\left\|u_{0}\right\|_{L^{2}} \lesssim 1, \\
T^{\frac{3}{2}-b-b_{1}-c_{1}}\left\|\left(n_{0}, n_{1}\right)\right\|_{\mathcal{W}} \lesssim 1 \\
T^{\frac{3}{2}-2 b_{1}-c}\left\|u_{0}\right\|_{L^{2}}^{2} \lesssim\left\|\left(n_{0}, n_{1}\right)\right\|_{\mathcal{W}}
\end{gathered}
$$

one obtains sufficient conditions for a contraction argument yielding the existence of a fixed point $u \in X_{0, b_{1}}^{S}, n_{ \pm} \in X_{-\frac{1}{2}, b}^{W \pm}$ of (3.2)-(3.3) such that

$$
\|u\|_{X_{0, b_{1}}^{S}} \lesssim T^{\frac{1}{2}-b_{1}}\left\|u_{0}\right\|_{L^{2}}, \quad\left\|n_{ \pm}\right\|_{X_{-\frac{1}{2}, b}^{W^{ \pm}}} \lesssim T^{\frac{1}{2}-b}\left\|\left(n_{0}, n_{1}\right)\right\|_{\mathcal{W}}
$$

Similarly estimating (3.2) in $C\left([0, T] ; L_{x}^{2}\right)$ by applying Lemmas 2.1(国), 2.3(可) and (3.6) shows that in fact $u \in C\left([0, T] ; L_{x}^{2}\right)$. We may therefore invoke the conservation law (1.2) to conclude $\|u(T)\|_{L_{r}^{2}}=\left\|u_{0}\right\|_{L^{2}}$, and thus we are concerned only with the possibility of growth in $\|n(t)\|_{\mathcal{W}}$ from one time step to the next. Suppose that after some number of iterations we reach a time where $\|n(t)\| \mathcal{W} \gg\|u(t)\|_{L_{x}^{2}}^{2}=\left\|u_{0}\right\|_{L_{x}^{2}}^{2}$. Take this time position as the initial time $t=0$ so that $\left\|u_{0}\right\|_{L^{2}}^{2} \ll\left\|\left(n_{0}, n_{1}\right)\right\|_{\mathcal{W}}$. Then (3.5) is automatically satisfied, and by (3.4) we may select a time increment of size

$$
T \sim\left\|\left(n_{0}, n_{1}\right)\right\|_{\mathcal{W}}^{-1 /\left(\frac{3}{2}-b-b_{1}-c_{1}\right)}=\left\|\left(n_{0}, n_{1}\right)\right\|_{\mathcal{W}}^{-2}
$$

where the right-hand side follows by selecting the optimal condition $b+b_{1}+c_{1}=1$ in Lemma 3.1(国). Since

$$
n=W\left(n_{0}, n_{1}\right)+W *_{R}\left(\partial_{x}|u|^{2}\right)
$$

we can apply Lemmas 2.1(b), 2.3(b) and follow through with (3.6) to obtain

$$
\begin{aligned}
\|n(T)\|_{\mathcal{W}} & \leq(1+T)\left\|\left(n_{0}, n_{1}\right)\right\|_{\mathcal{W}}+C T^{\frac{3}{2}-\left(2 b_{1}+c\right)}\left\|u_{0}\right\|_{L^{2}}^{2} \\
& \leq\left\|\left(n_{0}, n_{1}\right)\right\|_{\mathcal{W}}+C T^{\frac{1}{2}}\left(\left\|u_{0}\right\|_{L^{2}}^{2}+1\right)
\end{aligned}
$$


where $C$ is some fixed constant. The second line above follows by selecting the optimal condition $2 b_{1}+c=1$ in Lemma 3.1(b), and using (3.7) to obtain $T\left\|\left(n_{0}, n_{1}\right)\right\|_{\mathcal{W}}$ $\leq C T^{\frac{1}{2}}$. From this we see that we can carry out $m$ iterations on time intervals, each of length (3.7), where

$$
m \sim \frac{\left\|\left(n_{0}, n_{1}\right)\right\| \mathcal{W}}{T^{\frac{1}{2}}\left(\left\|u_{0}\right\|_{L^{2}}^{2}+1\right)}
$$

before the quantity $\|n(t)\|_{\mathcal{W}}$ doubles. The total time we advance after these $m$ iterations, by (3.7) and (3.8), is

$$
m T \sim \frac{1}{\left\|u_{0}\right\|_{L^{2}}^{2}+1}
$$

which is independent of $\|n(t)\|_{\mathcal{W}}$.

We can now repeat this entire procedure, each time advancing a time of length $\sim\left(\left\|u_{0}\right\|_{L^{2}}^{2}+1\right)^{-1}$ (independent of the size of $\left.\|n(t)\|_{\mathcal{W}}\right)$. Upon each repetition, the size of $\|n(t)\|_{\mathcal{W}}$ will at most double, giving the exponential-in-time upper bound stated in Theorem 1.1 .

\section{3-D KLEIN-GORDON-SChrÖDINGER SYSTEM}

The goal of this section is to prove Theorem 1.3. For the Klein-Gordon-Schrödinger system (1.4), no special multilinear estimates are needed. Instead, we will work in standard space-time norms and use Sobolev imbedding and the Hölder inequality.

We shall use the conservation law (1.5) to control the growth of $u(t)$ from one time step to the next and track the growth of $\|n(t)\|_{\mathcal{G}}$, where the $\mathcal{G}$ norm was defined in (2.3), by direct estimation. For expositional convenience, we restrict ourselves to dimension $d=3$ although similar results do hold for $d=1$ and $d=2$.

Proof of Theorem 1.3. (1.4) has the integral equation formulation

$$
\begin{aligned}
& u(t)=U(t) u_{0}+i U *_{R}[n u](t), \\
& n(t)=G(t)\left(n_{0}, n_{1}\right)+G *_{R}\left(|u|^{2}\right)(t) .
\end{aligned}
$$

Define the maps $\Lambda_{S}, \Lambda_{G}$ as

$$
\begin{aligned}
& \Lambda_{S}(u, n)=U u_{0}+i U *_{R}[n u], \\
& \Lambda_{G}(u)=G\left(n_{0}, n_{1}\right)+G *_{R}\left(|u|^{2}\right) .
\end{aligned}
$$

Let $\operatorname{Str}=L_{[0, T]}^{10 / 3} L_{x}^{10 / 3} \cap L_{[0, T]}^{8} L_{x}^{12 / 5}$. We seek a fixed point

$$
(u(t), n(t))=\left(\Lambda_{S}(u, n), \Lambda_{G}(u)\right)
$$

in the space $\left[C\left([0, T] ; L_{x}^{2}\right) \cap \operatorname{Str}\right] \times C\left([0, T] ; \mathcal{G}_{x}\right)$. Apply Lemma 2.3(可) with $(\tilde{q}, \tilde{r})=$ $\left(\frac{20}{9}, 5\right)$ for $d=3$ to obtain

$$
\left\|\Lambda_{S}(u, n)\right\|_{C\left([0, T] ; L_{x}^{2}\right) \cap \operatorname{Str}} \lesssim\left\|u_{0}\right\|_{L^{2}}+T^{1 / 4}\|n\|_{L_{[0, T]}^{\infty} L_{x}^{2}}\|u\|_{L_{[0, T]}^{\frac{10}{3}}} L_{x^{3}}^{\frac{10}{3}} .
$$

Estimate (4.2) in $C\left([0, T] ; \mathcal{G}_{x}\right)$ and apply Lemma 2.3 (C) followed by Sobolev imbedding to obtain

$$
\left\|\Lambda_{G}(u)\right\|_{C\left([0, T] ; \mathcal{G}_{x}\right)} \leq\left\|\left(n_{0}, n_{1}\right)\right\|_{\mathcal{G}}+c T^{3 / 4}\|u\|_{L_{[0, T]}^{8} L_{x}^{12 / 5}}^{2}
$$


where we estimated it as: $\left\||u|^{2}\right\|_{L_{[0, T]}^{1} H_{x}^{-1}} \lesssim\left\||u|^{2}\right\|_{L_{[0, T]}^{1} L_{x}^{6 / 5}} \leq T^{3 / 4}\|u\|_{L_{[0, T]}^{8} L_{x}^{12 / 5}}^{2}$. There are similar estimates for the differences $\Lambda_{S}\left(u_{1}, n_{1}\right)-\Lambda_{S}\left(u_{2}, n_{2}\right)$ and $\Lambda_{G}\left(u_{1}\right)-$ $\Lambda_{G}\left(u_{2}\right)$. If $T$ is such that

$$
\begin{gathered}
T^{1 / 4}\left\|u_{0}\right\|_{L^{2}} \lesssim 1, \\
T^{1 / 4}\left\|\left(n_{0}, n_{1}\right)\right\|_{\mathcal{G}} \lesssim 1, \\
T^{3 / 4}\left\|u_{0}\right\|_{L^{2}}^{2} \lesssim\left\|\left(n_{0}, n_{1}\right)\right\|_{\mathcal{G}},
\end{gathered}
$$

then a contraction argument implies there is a solution $(u, n)$ to (1.4) on $[0, T]$ such that

$$
\begin{aligned}
& \|u\|_{C\left([0, T] ; L_{x}^{2}\right) \cap S \operatorname{str}} \lesssim\left\|u_{0}\right\|_{L^{2}}, \\
& \|n\|_{C\left([0, T] ; \mathcal{G}_{x}\right)} \leq\left\|\left(n_{0}, n_{1}\right)\right\|_{\mathcal{G}}+c T^{3 / 4}\left\|u_{0}\right\|_{L^{2}}^{2} .
\end{aligned}
$$

By the conservation of mass, we have $\|u(t)\|_{L_{x}^{2}}=\left\|u_{0}\right\|_{L^{2}}$, and are thus concerned only with the possibility that $\|n(t)\|_{\mathcal{G}}$ grows excessively from one local increment to the next. Suppose that after some number of iterations $\|n(t)\|_{\mathcal{G}} \gg\|u(t)\|_{L^{2}}^{2}=$ $\left\|u_{0}\right\|_{L^{2}}^{2}$. Consider this time as the initial time so that $\left\|\left(n_{0}, n_{1}\right)\right\|_{\mathcal{G}} \gg\left\|u_{0}\right\|_{L^{2}}^{2}$. Then (4.5) is automatically satisfied, and by (4.4), we may thus take

$$
T \sim\left\|\left(n_{0}, n_{1}\right)\right\|_{\mathcal{G}}^{-4} .
$$

We see from (4.7) that, after $m$ iterations, each of size (4.8), where

$$
m \sim \frac{\left\|\left(n_{0}, n_{1}\right)\right\|_{\mathcal{G}}}{T^{3 / 4}\left\|u_{0}\right\|_{L^{2}}^{2}},
$$

the quantity $\|n(t)\|_{\mathcal{G}}$ at most doubles. The total time advanced after these $m$ iterations is

$$
m T \sim \frac{1}{\left\|u_{0}\right\|_{L^{2}}^{2}} .
$$

We can now repeat this entire procedure, each time advancing a time of length $\sim\left\|u_{0}\right\|_{L^{2}}^{-2}$ (independent of the size of $\|n(t)\|_{\mathcal{G}}$ ). Upon each repetition, the size of $\|n(t)\|_{\mathcal{G}}$ will at most double, giving the exponential-in-time upper bound stated in Theorem 1.3 .

\section{Appendix A. Proof of the multilinear estimates (expository)}

In this section, we prove Lemma 3.1. The material here is taken from [11, Lemmas $4.3,4.4$ with only a slight modification at one stage. This modification was described in a note under the heading "proof" following the statement of Lemma 3.1. Given its importance in our scheme, the full proof is included here in detail.

We need the calculus lemmas:

Lemma A.1 ([1], Lemma 4.1). Let $f \in L^{q}(\mathbb{R}), g \in L^{q^{\prime}}(\mathbb{R})$ for $1 \leq q, q^{\prime} \leq \infty$ and $\frac{1}{q}+\frac{1}{q^{\prime}}=1$. Assume that $f, g$ are nonnegative, even, and nonincreasing for a positive argument. Then $f * g$ enjoys the same properties.

Define

$$
[\lambda]_{+}= \begin{cases}\lambda & \text { if } \lambda>0 \\ \epsilon & \text { if } \lambda=0 \\ 0 & \text { if } \lambda<0\end{cases}
$$


Lemma A.2 ([1], Lemma 4.2). Let $0 \leq a_{-} \leq a_{+}$and $a_{+}+a_{-}>\frac{1}{2}$. Then $\forall s \in \mathbb{R}$,

$$
\int_{y}\langle y-s\rangle^{-2 a_{+}}\langle y+s\rangle^{-2 a_{-}} d y \leq c\langle s\rangle^{-\alpha}
$$

where $\alpha=2 a_{-}-\left[1-2 a_{+}\right]_{+}$.

Proof of Lemma 3.1(国). We shall only do the + case. The estimate is equivalent to

$$
|S| \leq c\|v\|_{2}\left\|v_{1}\right\|_{2}\left\|v_{2}\right\|_{2}
$$

where

$$
S=\int_{*} \frac{\hat{v} \hat{v}_{1} \hat{v}_{2}\langle\xi\rangle^{1 / 2}}{\langle\sigma\rangle^{b}\left\langle\sigma_{1}\right\rangle^{c_{1}}\left\langle\sigma_{2}\right\rangle^{b_{1}}}
$$

with $\hat{v}=\hat{v}(\xi, \tau), \hat{v}_{1}=\hat{v}_{1}\left(\xi_{1}, \tau_{1}\right), \hat{v}_{2}=\hat{v}_{2}\left(\xi_{2}, \tau_{2}\right), \sigma_{1}=\tau_{1}+\xi_{1}^{2}, \sigma_{2}=\tau_{2}+\xi_{2}^{2}$, $\sigma=\tau+\xi$, and $*$ indicates the restriction $\xi_{1}=\xi+\xi_{2}, \tau_{1}=\tau+\tau_{2}$. Indeed, for $\hat{v}_{1} \in L^{2}$,

$$
\begin{aligned}
& \int_{\xi_{1}, \tau_{1}} \widehat{n_{+} u}\left(\xi_{1}, \tau_{1}\right)\left\langle\sigma_{1}\right\rangle^{-c_{1}} \hat{v}_{1}\left(\xi_{1}, \tau_{1}\right) d \xi_{1} d \tau_{1} \\
& \quad=\int_{\xi_{1}, \tau_{1}}\left[\int_{\substack{\xi_{1}=\xi+\xi_{2} \\
\tau_{1}=\tau+\tau_{2}}} \hat{n}_{+}(\xi, \tau) \hat{u}\left(\xi_{2}, \tau_{2}\right)\right]\left\langle\sigma_{1}\right\rangle^{-c_{1}} \hat{v}_{1}\left(\xi_{1}, \tau_{1}\right) d \xi_{1} d \tau_{1} .
\end{aligned}
$$

Let $\hat{v}(\xi, \tau)=\hat{n}_{+}(\xi, \tau)\langle\xi\rangle^{-1 / 2}\langle\sigma\rangle^{b}$ and $\hat{v}_{2}\left(\xi_{2}, \tau_{2}\right)=\hat{u}\left(\xi_{2}, \tau_{2}\right)\left\langle\sigma_{2}\right\rangle^{b_{1}}$ to obtain A.1).

We note here that

$$
\begin{aligned}
\sigma_{1}- & \sigma-\sigma_{2}=\tau_{1}+\xi_{1}^{2}-(\tau+\xi)-\left(\tau_{2}+\xi_{2}^{2}\right) \\
& =\xi_{1}^{2}-\xi-\xi_{2}^{2} \\
& =\left(\xi_{1}-\frac{1}{2}\right)^{2}-\left(\xi_{2}-\frac{1}{2}\right)^{2} .
\end{aligned}
$$

In the case when $|\xi| \leq 1$, it suffices to estimate

$$
\begin{aligned}
\int_{\xi_{1}, \tau_{1}} & \int_{\substack{\xi_{1}=\xi+\xi_{2}=\tau+\tau_{2} \\
\tau_{1}=\tau}} \frac{\hat{v} \hat{v}_{1} \hat{v}_{2}}{\langle\sigma\rangle^{1 / 4}\left\langle\sigma_{1}\right\rangle^{1 / 4+}\left\langle\sigma_{2}\right\rangle^{1 / 4+}} \\
& =\int_{\xi_{1}, \tau_{1}}\left(\frac{\hat{v}_{1}}{\left\langle\sigma_{1}\right\rangle^{1 / 4+}}\right)\left(\int_{\substack{\xi_{1}=\xi+\xi_{2} \\
\tau_{1}=\tau+\tau_{2}}} \frac{\hat{v} \hat{v}_{2}}{\langle\sigma\rangle^{1 / 4}\left\langle\sigma_{2}\right\rangle^{1 / 4+}} d \xi_{2} d \tau_{2}\right) d \xi_{1} d \tau_{1} \\
& =\int_{x_{1}, t_{1}}\left[\frac{\hat{v}_{1}}{\left\langle\sigma_{1}\right\rangle^{1 / 4+}}\right]^{\vee}\left[\int_{\substack{\xi=\xi_{1}+\xi_{2} \\
\tau=\tau_{1}+\tau_{2}}} \frac{\hat{v} \hat{v}_{2}}{\langle\sigma\rangle^{1 / 4}\left\langle\sigma_{2}\right\rangle^{1 / 4+}} d \xi_{2} d \tau_{2}\right]^{\vee} \\
& =\int_{x, t}\left[\frac{\hat{v}_{1}}{\left\langle\sigma_{1}\right\rangle^{1 / 4+}}\right]^{\vee}\left[\frac{\hat{v}}{\langle\sigma\rangle^{1 / 4}}\right]^{\vee}\left[\frac{\hat{v}_{2}}{\left\langle\sigma_{2}\right\rangle^{1 / 4+}}\right]^{\vee}\left\|\left[\frac{\hat{v}_{2}}{\left\langle\hat{v}_{1}\right.}\right]^{\vee}\right\|_{\left.L_{t}\right\rangle^{1 / 4+}}^{\vee}\left\|^{\vee / 3}\right\|_{L_{t}^{8 / 3} L_{x}^{4}} .
\end{aligned}
$$


Now

$$
\begin{aligned}
& \left\|\left[\frac{\hat{v}}{\langle\sigma\rangle^{1 / 4}}\right]^{\vee}\right\|_{L_{t}^{4} L_{x}^{2}}=\left\|\int_{\xi} e^{i x \xi} \int_{\tau} e^{i t \tau} \frac{\hat{v}(\xi, \tau)}{\langle\tau+\xi\rangle^{1 / 4}} d \tau d \xi\right\|_{L_{t}^{4} L_{x}^{2}} \\
& \quad=\left\|\int_{\tau} e^{i t \tau} \frac{\hat{v}(\xi, \tau)}{\langle\tau+\xi\rangle^{1 / 4}} d \tau\right\|_{L_{t}^{4} L_{\xi}^{2}} \\
& \quad \leq\left\|\int_{\tau} e^{i t \tau} \frac{\hat{v}(\xi, \tau)}{\langle\tau+\xi\rangle^{1 / 4}} d \tau\right\|_{L_{\xi}^{2} L_{t}^{4}} \text { by Minkowskii } \\
& \quad \leq\left\|\int_{\tau} e^{i t \tau} \frac{\hat{v}(\xi, \tau-\xi)}{\langle\tau\rangle^{1 / 4}} d \tau\right\|_{L_{\xi}^{2} L_{t}^{4}} \text { by Sobolev } \\
& \quad \leq\left\|\int_{\tau} e^{i t \tau} \hat{v}(\xi, \tau-\xi) d \tau\right\|_{L_{\xi}^{2} L_{t}^{2}} \\
& \quad=\|\hat{v}\|_{L_{\xi}^{2} L_{\tau}^{2}}=\|v\|_{L^{2} .}
\end{aligned}
$$

For $j=1,2$, the estimate on

$$
\left\|\left[\frac{\hat{v}_{j}}{\left\langle\sigma_{j}\right\rangle^{1 / 4+}}\right]^{\vee}\right\|_{L_{t}^{8 / 3} L_{x}^{4}}
$$

is obtained by interpolating halfway between $\left\|\left[\left\langle\sigma_{j}\right\rangle^{-d} \hat{v}_{j}\right]^{\vee}\right\|_{L_{t}^{4} L_{x}^{\infty}} \leq\left\|v_{j}\right\|_{L_{t}^{2} L_{x}^{2}}$ for $d>\frac{1}{2}$ (Strichartz) and $\left\|\left[\hat{v}_{j}\right]^{\vee}\right\|_{L_{t}^{2} L_{x}^{2}}=\left\|v_{j}\right\|_{L_{t}^{2} L_{x}^{2}}$. This leaves us to estimate

$$
S^{\prime}=\int_{*} \frac{\hat{v} \hat{v}_{1} \hat{v}_{2}|\xi|^{1 / 2}}{\langle\sigma\rangle^{b}\left\langle\sigma_{1}\right\rangle^{c_{1}}\left\langle\sigma_{2}\right\rangle^{b_{1}}}
$$

with $\hat{v}=\hat{v}(\xi, \tau), \hat{v}_{1}=\hat{v}_{1}\left(\xi_{1}, \tau_{1}\right), \hat{v}_{2}=\hat{v}_{2}\left(\xi_{2}, \tau_{2}\right), \sigma_{1}=\tau_{1}+\xi_{1}^{2}, \sigma_{2}=\tau_{2}+\xi_{2}^{2}$, $\sigma=\tau+\xi$, and $*$ indicates the restriction $\xi_{1}=\xi+\xi_{2}, \tau_{1}=\tau+\tau_{2}$.

$\underline{\text { Region } \sigma \text { dominant, }|\sigma| \geq \max \left(\left|\sigma_{1}\right|,\left|\sigma_{2}\right|\right) \text {. }}$

$$
\begin{aligned}
S^{\prime} \leq & \left(\int_{\xi, \sigma}|\hat{v}|^{2}\right)^{1 / 2}\left(\int_{\xi, \sigma}\langle\sigma\rangle^{-2 b}\left|\int_{\xi_{2}, \sigma_{2}} \frac{\left|\hat{v}_{1} \hat{v}_{2}\right||\xi|^{1 / 2}}{\left\langle\sigma_{1}\right\rangle^{c_{1}}\left\langle\sigma_{2}\right\rangle^{b_{1}}}\right|^{2}\right)^{1 / 2} \\
& \leq\left(\int_{\xi, \sigma}|\hat{v}|^{2}\right)^{1 / 2}\left(\int_{\xi, \sigma}\langle\sigma\rangle^{-2 b}\left(\int_{\xi_{2}, \sigma_{2}}\left|\hat{v}_{1} \hat{v}_{2}\right|^{2}\right)\left(\int_{\xi_{2}, \sigma_{2}} \frac{|\xi|}{\langle\sigma\rangle^{2 c_{1}}\left\langle\sigma_{2}\right\rangle^{2 b_{1}}}\right)\right)^{1 / 2} \\
\text { (A.3) } \leq & \left(\sup _{\sigma, \xi}\langle\sigma\rangle^{-2 b} \int_{\sigma_{2}} \int_{\xi_{2}} \frac{|\xi|}{\left\langle\sigma_{1}\right\rangle^{2 c_{1}}\left\langle\sigma_{2}\right\rangle^{2 b_{1}}} d \xi_{2} d \sigma_{2}\right)^{1 / 2}\|v\|_{2}\left\|v_{1}\right\|_{2}\left\|v_{2}\right\|_{2} .
\end{aligned}
$$

The inner integral is taken over fixed $\sigma, \xi, \sigma_{2}$. Since $\xi_{1}=\xi+\xi_{2}$, we have $d \xi_{1}=d \xi_{2}$, and since $\sigma_{1}-\sigma-\sigma_{2}=\left(\xi_{1}-\frac{1}{2}\right)^{2}-\left(\xi_{2}-\frac{1}{2}\right)^{2}$, we have

$$
d \sigma_{1}=\left[+2\left(\xi_{1}-\frac{1}{2}\right)-2\left(\xi_{2}-\frac{1}{2}\right)\right] d \xi_{1}=2 \xi d \xi_{2} .
$$


Thus, the quantity in parentheses in (A.3) is bounded by

$$
\begin{aligned}
\sup _{\sigma}\langle\sigma\rangle^{-2 b} \int_{\sigma_{2}} \int_{\sigma_{1}} \frac{d \sigma_{1} d \sigma_{2}}{\left\langle\sigma_{1}\right\rangle^{2 c_{1}}\left\langle\sigma_{2}\right\rangle^{2 b_{1}}} \\
\quad \leq \sup _{\sigma}\langle\sigma\rangle^{-2 b}\langle\sigma\rangle^{\left[1-2 c_{1}\right]_{+}}\langle\sigma\rangle^{\left[1-2 b_{1}\right]_{+}} \\
\quad \leq \sup _{\sigma}\langle\sigma\rangle^{-2 b+\left[1-2 c_{1}\right]_{+}+\left[1-2 b_{1}\right]_{+}}
\end{aligned}
$$

since $\sigma$ is dominant. If $b, c_{1}, b_{1}<\frac{1}{2}$, then the exponent is $2-2 b-2 c_{1}-2 b_{1}$, and it suffices to have $b+c_{1}+b_{1} \geq 1$.

Region $\sigma_{1}$ dominant, $\left|\sigma_{1}\right| \geq \max \left(|\sigma|,\left|\sigma_{2}\right|\right)$.

By the Cauchy-Schwarz method, it suffices to show

$$
\sup _{\xi_{1}, \sigma_{1}}\left\langle\sigma_{1}\right\rangle^{-2 c_{1}} \int_{\sigma_{2}} \int_{\xi_{2}}|\xi|\langle\sigma\rangle^{-2 b}\left\langle\sigma_{2}\right\rangle^{-2 b_{1}} d \xi_{2} d \sigma_{2}
$$

is finite.

Subregion $\left|\xi_{1}-\frac{1}{2}\right| \leq 2\left|\xi_{2}-\frac{1}{2}\right|$. Then $|\xi| \leq 3\left|\xi_{2}-\frac{1}{2}\right|$. The inner integral over $\xi_{2}$ is taken with $\sigma_{1}, \xi_{1}, \sigma_{2}$ fixed. Since $\sigma_{1}-\sigma-\sigma_{2}=\left(\xi_{1}-\frac{1}{2}\right)^{2}-\left(\xi_{2}-\frac{1}{2}\right)^{2}$, we have $d \sigma=2\left(\xi_{2}-\frac{1}{2}\right) d \xi_{2}$ and thus (A.4) is bounded by

$$
\begin{gathered}
\sup _{\sigma_{1}}\left\langle\sigma_{1}\right\rangle^{-2 c_{1}} \int_{\sigma_{2}=0}^{\left|\sigma_{1}\right|}\left\langle\sigma_{2}\right\rangle^{-2 b_{1}} d \sigma_{2} \int_{\sigma=0}^{\left|\sigma_{1}\right|}\langle\sigma\rangle^{-2 b} d \sigma \\
\leq \sup _{\sigma_{1}}\left\langle\sigma_{1}\right\rangle^{-2 c_{1}+\left[1-2 b_{1}\right]_{+}+[1-2 b]_{+}} .
\end{gathered}
$$

If $b, b_{1}<\frac{1}{2}$, then the exponent here is $2-2 b_{1}-2 b-2 c_{1}$, and thus we need $b_{1}+b+c_{1} \geq 1$.

Subregion $\left|\xi_{1}-\frac{1}{2}\right| \geq 2\left|\xi_{2}-\frac{1}{2}\right|$. Since $\xi=\xi_{1}-\xi_{2}$, we have $|\xi| \leq \frac{3}{2}\left|\xi_{1}-\frac{1}{2}\right|$. Also $\frac{3}{4}\left(\xi_{1}-\frac{1}{2}\right)^{2} \leq\left(\xi_{1}-\frac{1}{2}\right)^{2}-\left(\xi_{2}-\frac{1}{2}\right)^{2}=\sigma_{1}-\sigma_{2}-\sigma \leq 3\left|\sigma_{1}\right|$, and thus $\left(\xi_{1}-\frac{1}{2}\right)^{2} \leq 4\left|\sigma_{1}\right|$. Thus (A.4) is bounded by

$$
\sup _{\xi_{1}, \sigma_{1}}\left\langle\xi_{1}\right\rangle^{1-4 c_{1}} \int_{\sigma_{2}} \int_{\xi_{2}}\langle\sigma\rangle^{-2 b}\left\langle\sigma_{2}\right\rangle^{-2 b_{1}} d \xi_{2} d \sigma_{2}
$$

We change variables $y=\left(\xi_{2}-\frac{1}{2}\right)^{2}$ to obtain

$$
\sup _{\xi_{1}, \sigma_{1}}\left\langle\xi_{1}\right\rangle^{1-4 c_{1}} \int_{y=-\left\langle\xi_{1}\right\rangle^{2}}^{y=\left\langle\xi_{1}\right\rangle^{2}}|y|^{-1 / 2} \int_{\sigma_{2}}\langle\sigma\rangle^{-2 b}\left\langle\sigma_{2}\right\rangle^{-2 b_{1}} d \sigma_{2} d y .
$$

The inner integral over $\sigma_{2}$ is taken with fixed $y=\left(\xi_{2}-\frac{1}{2}\right)^{2}, \xi_{1}, \sigma_{1}$, and thus $\sigma_{2}+\sigma=\sigma_{1}-\left(\xi_{1}-\frac{1}{2}\right)^{2}-\left(\xi_{2}-\frac{1}{2}\right)^{2}=\sigma_{1}-\left(\xi_{1}-\frac{1}{2}\right)^{2}-y$ is fixed. By Lemma A.2.

$$
\int_{\sigma_{2}}\langle\sigma\rangle^{-2 b}\left\langle\sigma_{2}\right\rangle^{-2 b_{1}} d \sigma_{2} \leq c\left\langle\sigma_{1}-\left(\xi_{1}-\frac{1}{2}\right)^{2}-y\right\rangle^{-\alpha} \quad \text { if } b_{1}+b>\frac{1}{2}
$$


where $\alpha=2 b-\left[1-2 b_{1}\right]_{+}$if $b_{1} \geq b$ or $=2 b_{1}-[1-2 b]_{+}$if $b \geq b_{1}$. By Lemma A.1 with $f(y)=\chi_{-\left\langle\xi_{1}\right\rangle^{2} \leq y \leq\left\langle\xi_{1}\right\rangle^{2}}(y)|y|^{-1 / 2}$ and $g(y)=\langle y\rangle^{-\alpha}$,

$$
\begin{aligned}
& \sup _{\sigma_{1}} \int_{y=-\left\langle\xi_{1}\right\rangle^{2}}^{+\left\langle\xi_{1}\right\rangle^{2}}|y|^{-1 / 2}\left\langle\sigma_{1}-\left(\xi_{1}-\frac{1}{2}\right)^{2}-y\right\rangle^{-\alpha} d y \\
& \quad \leq \int_{y=-\left\langle\xi_{1}\right\rangle^{2}}^{+\left\langle\xi_{1}\right\rangle^{2}}|y|^{-1 / 2}\langle y\rangle^{-\alpha} d y \\
& \quad \leq\left\langle\xi_{1}\right\rangle^{[1-2 \alpha]_{+}}
\end{aligned}
$$

and hence (A.5) is controlled by

$$
\sup _{\xi_{1}}\left\langle\xi_{1}\right\rangle^{1-4 c_{1}+[1-2 \alpha]_{+}} .
$$

We now consider the exponent. Suppose $b, b_{1}<\frac{1}{2}$ but $b_{1}+b>\frac{1}{2}$. Then $\alpha=$ $-1+2 b+2 b_{1}$.

Case 1. $\alpha>\frac{1}{2} \Longleftrightarrow b+b_{1}>\frac{3}{4}$. Then we need $c_{1} \geq \frac{1}{4}$.

Case 2. $\alpha=\frac{1}{2} \Longleftrightarrow b+b_{1}=\frac{3}{4}$. Then we need $c_{1}>\frac{1}{4}$.

Case 3. $\alpha<\frac{1}{2} \Longleftrightarrow b+b_{1}<\frac{3}{4}$. Then the exponent is $4-4 c_{1}-4 b-4 b_{1}$, and we need $b+b_{1}+c_{1} \geq 1$.

Region $\sigma_{2}$ dominant, $\left|\sigma_{2}\right| \geq \max \left(|\sigma|,\left|\sigma_{1}\right|\right)$. This is analogous to the $\sigma_{1}$ dominant case, but we carry it out anyway. By the Cauchy-Schwarz method, need to show

$$
\sup _{\xi_{2}, \sigma_{2}}\left\langle\sigma_{2}\right\rangle^{-2 b_{1}} \iint_{\sigma_{1}, \xi_{1}}|\xi|\langle\sigma\rangle^{-2 b}\left\langle\sigma_{1}\right\rangle^{-2 c_{1}} d \sigma_{1} d \xi_{1}
$$

is finite.

Subregion $\left|\xi_{2}-\frac{1}{2}\right| \leq 2\left|\xi_{1}-\frac{1}{2}\right|$. Then $|\xi| \leq 3\left|\xi_{1}-\frac{1}{2}\right|$. The inner integral over $\xi_{1}$ is taken with $\sigma_{1}, \xi_{2}, \sigma_{2}$ fixed. Since $\sigma_{1}-\sigma-\sigma_{2}=\left(\xi_{1}-\frac{1}{2}\right)^{2}-\left(\xi_{2}-\frac{1}{2}\right)^{2}$, we have $-d \sigma=2\left(\xi_{1}-\frac{1}{2}\right) d \xi_{1}$. Thus (A.6) is bounded by

$$
\begin{gathered}
\sup _{\sigma_{2}}\left\langle\sigma_{2}\right\rangle^{-2 b_{1}} \int_{\sigma_{1}}\left\langle\sigma_{1}\right\rangle^{-2 c_{1}} d \sigma_{1} \int_{\sigma}\langle\sigma\rangle^{-2 b} d \sigma \\
\leq \sup _{\sigma_{2}}\left\langle\sigma_{2}\right\rangle^{-2 b_{1}+\left[1-2 c_{1}\right]_{+}+[1-2 b]_{+}} .
\end{gathered}
$$

If $c_{1}, b<\frac{1}{2}$, then we need $b_{1}+c_{1}+b \geq 1$.

Subregion $\left|\xi_{2}-\frac{1}{2}\right| \geq 2\left|\xi_{1}-\frac{1}{2}\right|$. Then $|\xi| \leq \frac{3}{2}\left|\xi_{2}-\frac{1}{2}\right|$. Also, $\frac{3}{4}\left(\xi_{2}-\frac{1}{2}\right)^{2} \leq$ $\left(\xi_{2}-\frac{1}{2}\right)^{2}-\left(\xi_{1}-\frac{1}{2}\right)^{2}=-\sigma_{1}+\sigma+\sigma_{2} \leq 3\left|\sigma_{2}\right|$ and hence $\left(\xi_{2}-\frac{1}{2}\right)^{2} \leq 4\left|\sigma_{2}\right|$. We change variable $y=\left(\xi_{1}-\frac{1}{2}\right)^{2}$ to obtain that (A.6) is bounded by

$$
\sup _{\sigma_{2}, \xi_{2}}\left\langle\xi_{2}\right\rangle^{1-4 b_{1}} \int_{y=-\left\langle\xi_{2}\right\rangle^{2}}^{\left\langle\xi_{2}\right\rangle^{2}}|y|^{-1 / 2} \int_{\sigma_{1}}\langle\sigma\rangle^{-2 b}\left\langle\sigma_{1}\right\rangle^{-2 c_{1}} d \sigma_{1} d y .
$$

Since $-\sigma+\sigma_{1}=\sigma_{2}+\left(\xi_{1}-\frac{1}{2}\right)^{2}-\left(\xi_{2}-\frac{1}{2}\right)^{2}=\sigma_{2}-\left(\xi_{2}-\frac{1}{2}\right)^{2}+y$ is fixed, by Lemma A.2.

$$
\int_{\sigma_{1}}\langle\sigma\rangle^{-2 b}\left\langle\sigma_{1}\right\rangle^{-2 c_{1}} d \sigma_{1} \leq\left\langle\sigma_{2}-\left(\xi_{2}-\frac{1}{2}\right)^{2}+y\right\rangle^{-\alpha} \quad \text { if } b+c_{1}>\frac{1}{2}
$$


with $\alpha=2 b-\left[1-2 c_{1}\right]_{+}$if $c_{1} \geq b$ and $\alpha=2 c-[1-2 b]_{+}$if $b \geq c_{1}$. By Lemma A.1 with $f(y)=\chi_{-\left\langle\xi_{2}\right\rangle^{2} \leq y \leq\left\langle\xi_{2}\right\rangle^{2}}(y)|y|^{-1 / 2}$ and $g(y)=\langle y\rangle^{-\alpha}$,

$$
\begin{aligned}
& \sup _{\sigma_{2}} \int_{y=-\left\langle\xi_{2}\right\rangle^{2}}^{\left\langle\xi_{2}\right\rangle^{2}}|y|^{-1 / 2}\left\langle\sigma_{2}-\left(\xi_{2}-\frac{1}{2}\right)^{2}+y\right\rangle^{-\alpha} d y \\
& \quad \leq \int_{y=-\left\langle\xi_{2}\right\rangle^{2}}^{\left\langle\xi_{2}\right\rangle^{2}}|y|^{-1 / 2}\langle y\rangle^{-\alpha} d y \\
& \quad \leq\left\langle\xi_{2}\right\rangle^{[1-2 \alpha]_{+}} .
\end{aligned}
$$

Hence (A.7) is bounded by

$$
\sup _{\xi_{2}}\left\langle\xi_{2}\right\rangle^{1-4 b_{1}+[1-2 \alpha]_{+}} .
$$

We now consider the exponent. If $b, c_{1}<\frac{1}{2}$ and $b+c_{1}>\frac{1}{2}$, then $\alpha=-1+2 b+2 c_{1}$. Case 1. $\alpha<\frac{1}{2} \Longleftrightarrow b+c_{1}<\frac{3}{4}$. Then the exponent is $4-4 b_{1}-4 b-4 c_{1}$ so we need $b+b_{1}+c_{1} \geq 1$.

Case 2. $\alpha=\frac{1}{2} \Longleftrightarrow b+c_{1}=\frac{3}{4}$. Here, we need $b_{1}>\frac{1}{4}$.

Case 3. $\alpha>\frac{1}{2} \Longleftrightarrow b+c_{1}>\frac{3}{4}$. Here, we need $b_{1} \geq \frac{1}{4}$.

Proof of Lemma 3.1 (b). We show that the proof of Lemma 3.1 (b) is actually identical to that of Lemma 3.1 (国). We discuss only the + case. The estimate is equivalent to showing

$$
|W| \leq c\|v\|_{2}\left\|v_{1}\right\|_{2}\left\|v_{2}\right\|_{2}
$$

where

$$
W=\int_{*} \frac{\hat{v} \hat{v}_{1} \hat{v}_{2}|\xi|\langle\xi\rangle^{-1 / 2}}{\langle\sigma\rangle^{c}\left\langle\sigma_{1}\right\rangle^{b_{1}}\left\langle\sigma_{2}\right\rangle^{b_{1}}}
$$

with $\hat{v}=\hat{v}(\xi, \tau), \hat{v}_{1}=\hat{v}_{1}\left(\xi_{1}, \tau_{1}\right), \hat{v}_{2}\left(\xi_{2}, \tau_{2}\right), \sigma=\tau+\xi, \sigma_{1}=\tau_{1}+\xi_{1}^{2}, \sigma_{2}=\tau_{2}+\xi_{2}^{2}$, and $*$ indicates the restriction $\xi=\xi_{1}-\xi_{2}, \tau=\tau_{1}-\tau_{2}$. Indeed, for $\hat{v} \in L^{2}$,

$$
\begin{aligned}
\int_{\xi, \tau} & {\left[\partial_{x}\left(u_{1} \bar{u}_{2}\right)\right] \uparrow(\xi, \tau)\langle\xi\rangle^{-1 / 2}\langle\sigma\rangle^{-c} \hat{v}(\xi, \tau) d \xi d \tau } \\
& =\int_{\xi, \tau}|\xi|\left[\int_{\substack{\xi=\xi_{1}-\xi_{2} \\
\tau=\tau_{1}-\tau_{2}}} \hat{u}_{1}\left(\xi_{1}, \tau_{1}\right) \hat{u}_{2}\left(\xi_{2}, \tau_{2}\right)\right]\langle\xi\rangle^{-1 / 2}\langle\sigma\rangle^{-c} \hat{v}(\xi, \tau) d \xi d \tau .
\end{aligned}
$$

Set $\hat{v}_{1}\left(\xi_{1}, \tau_{1}\right)=\hat{u}_{1}\left(\xi_{1}, \tau_{1}\right)\left\langle\sigma_{1}\right\rangle^{b_{1}}, \hat{v}_{2}\left(\xi_{2}, \tau_{2}\right)=\hat{u}_{2}\left(\xi_{2}, \tau_{2}\right)\left\langle\sigma_{2}\right\rangle^{b_{1}}$ to obtain (A.8).

We note that (A.8) is the same as (A.1) considered in the proof of Lemma 3.1 (国) with $b, c_{1}$ in (A.1) replaced by $c, b_{1}$, respectively. Thus, the condition $b+b_{1}+c_{1} \geq 1$ in Lemma 3.1 (回) becomes $c+2 b_{1} \geq 1$ in Lemma 3.1 (b).

\section{REFERENCES}

[1] Takafumi, Akahori, Global solutions of the wave-Schrödinger system with rough data, Commun. Pure Appl. Anal. 4 (2005), no. 2, 209-240.

[2] Daniella Bekiranov, Takayoshi Ogawa, and Gustavo Ponce, Weak solvability and wellposedness of a coupled Schrödinger-Korteweg de Vries equation for capillary-gravity wave interactions, Proc. Amer. Math. Soc. 125 (1997), no. 10, 2907-2919. MR1403113 (97m:35238)

[3] Daniella Bekiranov, Takayoshi Ogawa, and Gustavo Ponce, Interaction equations for short and long dispersive waves, J. Funct. Anal. 158 (1998), no. 2, 357-388. MR.1648479 (99i:35143)

[4] J. Bourgain and J. Colliander, On wellposedness of the Zakharov system, Internat. Math. Res. Notices (1996), no. 11, 515-546. MR.1405972(97h:35206) 
[5] J. Bourgain, Fourier transform restriction phenomena for certain lattice subsets and applications to nonlinear evolution equations. I. Schrödinger equations, Geom. Funct. Anal. 3 (1993), no. 2, 107-156.

[6] J. Bourgain, Refinements of Strichartz' inequality and applications to $2 D$-NLS with critical nonlinearity, Internat. Math. Res. Notices (1998), no. 5, 253-283. MR 1616917 (99f:35184)

[7] M. Christ, J. Colliander, and T. Tao, Ill-posedness for nonlinear Schrödinger and wave equations, arxiv.org preprint, http://www.arxiv.org/abs/math.AP/0311048.

[8] J. Colliander, M. Keel, G. Staffilani, H. Takaoka, and T. Tao, Almost conservation laws and global rough solutions to a nonlinear Schrödinger equation, Math. Res. Lett. 9 (2002), no. 5-6, 659-682. MR1906069 (2003j:35294)

[9] A.J. Corcho and F. Linares, Well-posedness for the Schrödinger-Korteweg-de Vries system, Trans. Amer. Math. Soc. 359 (2007), no. 9, 4089-4106. MR2309177

[10] L. Glangetas and F. Merle, Concentration properties of blow-up solutions and instability results for Zakharov equation in dimension two. II, Comm. Math. Phys. 160 (1994), no. 2, 349-389. MR.1262202 (95e:35196)

[11] J. Ginibre, Y. Tsutsumi, and G. Velo, On the Cauchy problem for the Zakharov system, J. Funct. Anal. 151 (1997), no. 2, 384-436. MR1491547 (2000c:35220)

[12] J. Holmer, Local ill-posedness for the 1D Zakharov system, Electron. J. Differential Equations, 2007, no. 24, 22 pp. (electronic). MR.2299578 (2007k:35465)

[13] M. Keel and T. Tao, Endpoint Strichartz estimates, Amer. J. Math. 120 (1998), no. 5, 955980.

[14] C. E. Kenig, G. Ponce, and L. Vega, A bilinear estimate with applications to the KdV equation, J. Amer. Math. Soc. 9 (1996), no. 2, 573-603. MR1329387 (96k:35159)

[15] - Quadratic forms for the 1-D semilinear Schrödinger equation, Trans. Amer. Math. Soc. 348 (1996), no. 8, 3323-3353. MR1357398(96j:35233)

[16] H. Pecher, Global well-posedness below energy space for the 1-dimensional Zakharov system, Internat. Math. Res. Notices (2001), no. 19, 1027-1056. MR.1857386 (2002j:35036)

[17] _ Global solutions of the Klein-Gordon-Schrödinger system with rough data, Differential Integral Equations 17 (2004), no. 1-2, 179-214. MR2035502

[18] _ Global solutions with infinite energy for the one-dimensional Zakharov system, Electron. J. Differential Equations (2005), No. 41, 18 pp. (electronic). MR2135252

[19] , Rough solutions of a Schrödinger-Benjamin-Ono system, Differential Integral Equations, 19 (2006), no. 5, 517-535. MR2235139 (2007h: 35319)

[20] - The Cauchy problem for a Schrödinger-Korteweg-de Vries system with rough data, Differential Integral Equations, 18 (2005), no. 10, 1147-1174. MR2162627 (2006j:35210)

[21] R. S. Strichartz, Restrictions of Fourier transforms to quadratic surfaces and decay of solutions of wave equations, Duke Math. J. 44 (1977), no. 3, 705-714.

[22] N. Tzirakis, The Cauchy problem for the Klein-Gordon-Schrödinger system in low dimensions below the energy space, Communications in PDE 30 (2005), no. 5-6, 605-641. $\operatorname{MR} 2153510(2006 \mathrm{k}: 35280)$

Department of Mathematics, University of Toronto, 40 St. George St., Toronto, Ontario, Canada M5S 2E4

Department of Mathematics, University of California, Berkeley, Berkeley, CaliFORNIA 94720

Department of Mathematics, University of Toronto, 40 St. George St., Toronto, Ontario, CANADA M5S 2E4

Current address: Department of Mathematics, University of Illinois, 1409 Green St., Urbana, Illinois 61801 